19 Revue d'histoire du XIXe siècle

Société d'histoire de la révolution de 1848 et des

révolutions du XIXe siècle

$61 \mid 2020$

Dans l'intimité de l'exil

Nathalie BRÉMAND (dir.), Bibliothèques en utopie. Les socialistes et la lecture au XIX ${ }^{e}$ siècle

Jérôme Lamy

\title{
OpenEdition
}

Journals

Édition électronique

URL : https://journals.openedition.org/rh19/7357

DOI : $10.4000 /$ rh 19.7357

ISSN : $1777-5329$

Éditeur

La Société de 1848

Édition imprimée

Date de publication : 1 décembre 2020

Pagination : 298-300

ISSN : 1265-1354

Référence électronique

Jérôme Lamy, « Nathalie BRÉMAND (dir.), Bibliothèques en utopie. Les socialistes et la lecture au

XIXe siècle », Revue d'histoire du XIXe siècle [En ligne], 61 | 2020, mis en ligne le 20 janvier 2021, consulté

le 20 août 2021. URL : http://journals.openedition.org/rh19/7357 ; DOl : https://doi.org/10.4000/

rh19.7357

Ce document a été généré automatiquement le 20 août 2021.

Tous droits réservés 


\section{Nathalie BRÉMAND (dir.), Bibliothèques en utopie. Les socialistes et la lecture au XIX siècle}

Jérôme Lamy

\section{RÉFÉRENCE}

Nathalie BRÉMAND (dir.), Bibliothèques en utopie. Les socialistes et la lecture au XIXe siècle, Villeurbanne, Presses de l'ENSIBB, 2020, 262 p., $20 €$.

1 Les premiers socialismes ont partie liée avec l'univers de l'imprimé. La diffusion des idées, l'exposition des alternatives au monde tel qu'il était, la contestation des régimes en place, toutes ces formes d'expression ont connu une diffusion éditoriale inédite au $\mathrm{XIX}$ siècle. L'ouvrage dirigé par Nathalie Brémand se propose d'explorer l'espace ouvert par le double mouvement de l'«expansion de la consommation du livre» (p. 7) et de «la naissance du socialisme» (p. 8). Il s'agit donc « d'étudier la place du livre et de la lecture dans les théories et les pratiques des premiers socialistes» (p. 9). L'ouvrage se découpe en trois parties, la première consacrée aux livres, à leurs usages et à leurs manipulations chez les socialistes du début du XIX ${ }^{e}$ siècle. La deuxième se centre sur les pratiques de lecture, et la troisième s'intéresse aux bibliothèques.

2 Edward Castleton et Anne-Sophie Chambost détaillent la constitution des bibliothèques de Pierre-Joseph Proudhon. L'auteur de Qu'est-ce que la propriété ? disposait, à la fin de sa vie, d'une bibliothèque de plus de 1200 ouvrages. Les conditions matérielles de la constitution de ce corpus sont essentielles. La «culture [de Proudhon] s'est faite en autodidacte » (p. 63) et, au cours de ses études « il avait cruellement éprouvé le manque de livres » (p. 64). Le passage par le secteur de l'imprimerie à Besançon lui permet, en revanche, de se frotter aux textes philosophiques et philologiques. Les bibliothèques publiques sont également une ressource importante pour Proudhon; il tient des «carnets" et des «cahiers» qui inventorient les «livres à lire» (p. 67). C'est «[à] 
partir du moment où sa notoriété se renforce dans les milieux des réformateurs sociaux et des économistes, [que] Proudhon commence à recevoir des livres qui lui permettent de constituer sa propre bibliothèque » (p. 68). Cependant, entre l'exil et la prison, la construction d'un fonds d'ouvrages à peu près stable semble difficile. Ce sont surtout les livres acquis à la fin de l'existence du penseur socialiste qui forment le gros de sa bibliothèque. Edward Castleton et Anne-Sophie Chambost évoquent avec précision les limites de leur enquête: difficile, en effet, de comprendre la logique exacte de constitution du corpus de Proudhon. Entre livres achetés et livres donnés, comment faire la différence? Les dédicaces aident à se faire une idée à ce sujet, et «[1]es marginalia [...] qui figurent dans certains livres de Proudhon sont un témoignage précieux sur la bibliothèque vécue du Franc-Comtois [...]» (p. 71). Ce fonds d'ouvrage a nourri la formation du philosophe autodidacte, elle a aussi constitué un point d'appui pour son propre parcours d'intellectuel.

3 Le cas de Jules Gay, fidèle du mouvement owéniste, est également intéressant pour saisir les dynamiques de formation des bibliothèques. Thomas Bouchet détaille l'activité de cet «[i]nsatiable bibliophile» (p. 77), qui est aussi un auteur prolifique. Travaillant chez plusieurs éditeurs, Gay fonde également des maisons d'édition. Ce qui motive cet activisme éditorial, c'est l'idéal d'une «propagande par le livre » (p. 82). En cohérence avec ses idéaux politiques, Jules Gay s'oppose au droit d'auteur ; il s'agit de permettre une diffusion large des textes.

4 La circulation des écrits socialistes permet d'interroger les pratiques de lecture. Dans un chapitre sur la lecture des penseurs radicaux "dans l'atelier» (p. 93), Fabrice Bensimon et François Jarrige recomposent cette diffusion spécifique des écrits émancipateurs au XIX ${ }^{e}$ siècle. La "circulation de l'information " continue de passer, dans les milieux populaires, "par le bouche-à-oreille» (p. 95). Dans les secteurs de l'artisanat, mais aussi de la paysannerie, les lectures publiques se multiplient. Au sein des ateliers, "dans les interstices des temps et des espaces productifs", la parole émancipatrice se répand (p. 100). En Grande-Bretagne, «le Northern Star [...], un grand journal populaire chartiste est un vecteur de politisation et d'éducation dans les milieux ouvriers » (p. 104). La collectivisation de la lecture permet également de faire baisser le coût d'achat du journal. Tous les secteurs industriels ne peuvent permettre la lecture en groupe dans les ateliers : il est nécessaire que l'activité ne requière pas de machines trop bruyantes. Bensimon et Jarrige soulignent à bon droit que cette lecture au sein de «l'atelier » constitue un moyen de " proclamer l'autonomie et la résistance au nouvel ordre disciplinaire de la fabrique qui s'installe progressivement et imparfaitement » (p. 108). En conclusion de leur chapitre, les auteurs insistent sur la diffusion de ce modèle de lecture collective sur le lieu de travail, de La Havane, vers le Mexique, mais aussi aux États-Unis.

5 Pour comprendre la façon dont s'opère « l'éducation par le livre » (p. 115) en direction du monde ouvrier, Isabelle Matamoros explore le travail de Flora Tristan. La socialiste et féministe française a mis au point un véritable «programme d'éducation par le livre devant mener lecteurs et lectrices à prendre conscience des inégalités et à se mobiliser contre elles» (p.115). L'enjeu est de déployer une capacité émancipatrice " par étapes et de manière pragmatique » (p. 119). Un corpus essentiel de lectures fournirait ainsi le socle d'une prise de conscience. De ce point de vue, «Flora Tristan rejoint les contempteurs de la mauvaise littérature dont elle partage le regard parfois misérabiliste sur l'état culturel de la classe ouvrière » (p. 120). Toutefois, elle écarte les 
pensums moraux et privilégie " une lecture politique» (p.122), avec les ouvrages de Buret, Frégier, Parent, Blanc, Proudhon ou encore Moreau. Ces lectures, potentiellement édificatrices, font "émerger une réflexion critique» (p.124). En 1843-1844, Flora Tristan réalise son Tour de France à la rencontre des ouvriers et des ouvrières. Elle défend son idée d'Union ouvrière et sa vision émancipatrice de la lecture.

6 La constitution des bibliothèques est un enjeu politique important pour les premiers socialistes. Ludovic Robert et Quentin Schwrank consacrent un chapitre à la façon dont cet espace particulier est décrit dans l'Encyclopédie nouvelle de Pierre Leroux et Jean Reynaud. L'article « Bibliothèque ", probablement rédigé par Jean-Edmond Tournachon de Montvéran, commence par « esquisser une histoire globale de la bibliothèque depuis les âges les plus reculés » (p. 181). L'auteur défend l'idée d'un « réseau de bibliothèques publiques " géré par l'État (p. 183). Ici le socialisme de l'Encyclopédie nouvelle (elle-même traversée de fortes tensions) rejoint le républicanisme.

7 Enfin, l'étude qu'Agnès Sandras consacre aux « crispations » concernant les « ouvrages socialistes dans les premières bibliothèques populaires» (p. 233) permet de saisir les ambivalences d'un pouvoir qui, notamment sous le Second Empire, ne peut ignorer les aspirations populaires pour la culture, mais se méfie des effets potentiels de sédition. La tentative, à Saint-Étienne, d'interdire les auteurs socialistes dans la bibliothèque populaire, témoigne d'une autonomie progressive de ces lieux d'émancipation.

L'ouvrage dirigé par Nathalie Brémand permet d'ébaucher une cartographie des foyers éditoriaux de la mouvance socialiste au début du XIXe siècle. La diversité des usages du livre, l'expansion de l'imprimé et la volonté de certaines actrices et certains acteurs de faire du livre le point d'appui pour une transformation politique radicale signalent des transformations profondes dans la manière d'envisager le mouvement d'émancipation. Insister sur la multiplicité des approches du politique par le livre constitue l'un des apports essentiels de Bibliothèques en utopie. 